\title{
Resveratrol protects mice from paraquat-induced lung injury: The important role of SIRT1 and NRF2 antioxidant pathways
}

\author{
SHENGQIN LI*, GUANGJU ZHAO*, LONGWANG CHEN, YINWEI DING, \\ JIE LIAN, GUANGLIANG HONG and ZHONGQIU LU \\ Emergency Department, The First Affiliated Hospital of Wenzhou Medical University, \\ Wenzhou, Zhejiang 325000, P.R. China
}

Received March 22, 2015; Accepted October 29, 2015

DOI: $10.3892 / \mathrm{mmr} .2015 .4710$

\begin{abstract}
Sirtuin 1 (SIRT1) acts via the deacetylation of a number of crucial transcription factors and has been implicated in various biological processes, including oxidative stress. Previous studies have indicated that nuclear factor, erythroid 2-like 2 (NRF2) is an effective target of antioxidant therapy for paraquat (PQ) poisoning. However, the association between SIRT1 and NRF2 and their effects in PQ-induced oxidative stress remains to be elucidated. The current study demonstrated that PQ exposure upregulated the expression of SIRT1 and NRF2 following 6- and 24-h exposure in the lungs of mice. However, long-term exposure to PQ significantly decreased the expression of SIRT1 and NRF2. Resveratrol is a SIRT1 activator, and strongly enhanced SIRT1 expression and attenuated the lung injury resulting from PQ exposure in the current study. Additionally, treatment with resveratrol upregulated the expression of NRF2 and glutathione, increased the activity of heme oxygenase-1, superoxide dismutase and catalase, but depleted the expression of malondialdehyde. The present results demonstrated that resveratrol reduced $\mathrm{PQ}$-induced oxidative stress and lung injury, potentially through the positive feedback signaling loop between SIRT1 and NRF2.
\end{abstract}

\section{Introduction}

Paraquat (1,1'-dimethyl-4,4'-bipyridinium dichloride; PQ) is an effective, widely used, nonselective herbicide. It is toxic to humans and is associated with high mortality, mainly as a consequence of respiratory failure (1). Upon entering the cell, $\mathrm{PQ}$ undergoes cyclic single-electron reduction/oxidation through

Correspondence to: Mr. Zhongqiu Lu, Emergency Department, The First Affiliated Hospital of Wenzhou Medical University, 2 Fuxuexiang Street, Nanbaixiang, Ouhai, Wenzhou, Zhejiang 325000, P.R. China

E-mail:1zq640815@163.com

*Contributed equally

Key words: resveratrol, paraquat, oxidative stress, sirtuin 1, nuclear factor, erythroid 2-like 2 its quaternary ammonium nitrogen atoms and bipyridyl ring, producing reactive oxygen species (ROS) and PQ radicals (2). Redox cycling is considered to serve an important role in the initiation of the lung damage and fibrosis resulting from PQ exposure (2,3). In view of this, antioxidant therapy is considered an important strategy for the treatment of PQ poisoning $(2,3)$.

Sirtuin 1 (SIRT1) is an NAD ${ }^{+}$-dependent deacetylase (4). SIRT1 regulates a wide variety of cellular processes by deacetylating histones and numerous other crucial transcription factors, including factors in control of ROS production (5). The nuclear factor, erythroid 2-like 2 (NRF2) transcription factor is a member of the cap ' $n$ ' collar basic-region leucine zipper transcription factors, and is considered an effective target for antioxidant therapy for PQ poisoning $(3,6)$. Previous studies have demonstrated that the SIRT1-mediated deacetylation of NRF2 terminated the transcription of antioxidant genes (7). By contrast, SIRT1 is known to protect cells from oxidative stress injury, and silencing its activity results in decreased NRF2 expression levels $(8,9)$. However, the association between SIRT1 and NRF2 and their effect in PQ-induced oxidative stress remains unclear.

Resveratrol (3,4',5-trihydroxystilbene; Res) is a natural polyphenol present in grapes, berries, peanuts and other plants (10). Previous studies have reported the beneficial effects of Res in numerous diseases, including cancer, cardiovascular diseases, ischemic injuries and acute poisoning (11-13). This wide range of biological effects may be explained in part by the antioxidant properties of Res, and the activation and expression of SIRT1 is postulated to be a key event in the pathophysiology of Res $(10,11)$. Based on these data, the current study investigated the effects of Res on PQ-induced oxidative stress and lung injury. Furthermore, the potential roles of SIRT1 and NRF2 in this progress were also illustrated.

\section{Materials and methods}

Animals and reagents. Male Institute of Cancer Research mice (age, 6-8 weeks; weight, 18-20 g) were provided by the Animal Experimental Center of Wenzhou Medical University (Wenzhou, China). The study was approved by the Laboratory Animal Ethics Committee of Wenzhou Medical University \& Laboratory Animal Centre of Wenzhou Medical University (Wenzhou, China). Animals were housed in a 
room with a 12-h light/dark cycle and allowed free access to tap water and standard laboratory food. The PQ and Res used in the experiment were purchased from Sigma-Aldrich (St. Louis, MO, USA). Rabbit anti-mouse SIRT1 monoclonal antibody (cat. no. 3931) and rabbit anti-mouse Nrf2 polyclonal antibody (cat. no. ab31163) were obtained from Cell Signaling Technology, Inc. (Beverly, MA, USA) and Abcam (Cambridge, MA, USA), respectively. Polyclonal mouse anti- $\beta$ actin antibody (cat. no. BS1002) was purchased from Bioworld Technology (St. Louis Park, MN, USA). Formaldehyde, Hematoxylin and Eosin (H\&E) Staining kit, agarose gel, SDS-PAGE and Nuclear and Cytoplasmic Protein Extraction kit (no. P0028) were purchased from Beyotime Institute of Biotechnology (Haimen, China)

Experimental design. The dose- and time-dependent effects of Res on SIRT1 expression and lung injury after PQ exposure were examined by two independent experiments. A) Mice were randomly divided into four groups as follows: i) The control group, mice received saline solution by intraperitoneal (i.p.) injection $(n=6)$; ii) The Res group, mice received Res (30 mg/kg i.p.; $\mathrm{n}=6$ ); iii) The PQ group, mice received PQ (20 mg/kg i.p.; $\mathrm{n=6}$ ); iv) The PQ + Res group, mice received PQ (20 mg/kg i.p.) and Res (15, 30 or $60 \mathrm{mg} / \mathrm{kg}$ i.p.; $\mathrm{n}=6$ for each condition). PQ was dissolved in saline solution and injected intraperitoneally in a single toxic dose of $20 \mathrm{mg} / \mathrm{kg}$ of body weight based on preliminary experiments. Mice were anesthetized with $50 \mathrm{mg} / \mathrm{kg}$ pentobarbital (Hanlim Pharm Co., Ltd., Seoul, Korea). Pulmonary samples were collected at $24 \mathrm{~h}$ subsequent to PQ injection. B) Mice were randomly divided into three groups as follows: i) Control group $(n=18)$; ii) The PQ group, mice received PQ (20 mg/kg i.p.; $\mathrm{n}=18)$; iii) The $\mathrm{PQ}+$ Res group, mice received PQ (20 mg/kg i.p.) and Res (30 mg/kg i.p.; $\mathrm{n}=18)$. Pulmonary samples were collected at 6,24 and $72 \mathrm{~h}$ subsequent to PQ exposure, and tissues from the same mice were used for histopathological, PCR and western blot analyses.

Histopathological assessment of pulmonary tissue. The lower lobes of the right lungs of six mice pre group were removed and then transferred to $4 \%$ formaldehyde for $24 \mathrm{~h}$. The lungs were embedded in paraffin (Leica Microsystems, Wetzlar, Germany), and 2-3 butterfly-shaped sections of 4- $\mu \mathrm{m}$ thickness were cut per sample using a microtome (Leica RM2016; Leica Microsystems) and placed on glass microscope slides stained with hematoxylin and eosin (H\&E) for histopathological analysis. Lung histopathology images were acquired using a microscope (Nikon Eclipse 80i; Nikon, Tokyo, Japan). The severity of lung injury was determined by a histopathologist blinded to the protocol design.

Wet/dry (W/D) weight ratio. To quantify the magnitude of pulmonary edema, the lung W/D weight ratio was evaluated. The middle lobe of right lung of six animals was excised and the wet weight was determined, following which the lung was placed in a drying oven at $60^{\circ} \mathrm{C}$ for $72 \mathrm{~h}$ to stabilize the dry weight. The ratio of W/D was calculated.

$R N A$ isolation and reverse transcription-semiquantitative polymerase chain reaction ( $R T$-sqPCR). The total RNA of the upper right lung lobe of six mice per group was extracted using TRIzol reagent (Invitrogen; Thermo Fisher Scientific, Inc., Waltham,
MA USA). The cDNA was prepared from $2 \mu \mathrm{g}$ RNA using a PCR master mix (Promega Corp., Madison, WI, USA). The primers used were as follows: SIRT1, F 5'-ACGCTGTGGCAG ATTGTTATTA-3' and R 5'-TTGAAGAATGGTCTTGGG TCTT-3'; NRF2, F 5'-ATTCTTTCAGCAGCATCCTCTC-3' and R 5'-ACACTTCCAG GGGCACTATCTA-3', resulting in PCR products of 278 and $403 \mathrm{bp}$, respectively. The primers for mouse $\beta$-actin were F 5'-ATATCGCTGCGCTGGTCGTC-3' and R 5'-AGGATGGCGTGAGGGAGAGC-3', resulting in a PCR product of $517 \mathrm{bp}$. Amplified fragments of expected size were analyzed using a $2 \%$ agarose gel and images were captured under ultraviolet (UV) light (302 nm) (Tianneng Co., Shanghai, China). Gels were imaged using the ChemiDoc ${ }^{\mathrm{TM}}$ MP Imaging System (Bio-Rad Laboratories, Inc., Hercules, CA, USA) and quantified by densitometry using Quantity One 4.52 software (Bio-Rad Laboratories, Inc.). Data are presented as fold changes in gene expression normalized to $\beta$-actin.

Western blot analysis. The upper parts of the left lung from six animals per group were harvested and homogenized immediately. Total proteins were extracted from the lungs using the Nuclear and Cytoplasmic Protein Extraction kit according to the manufacturer's protocol. Equal amounts of total proteins $(40 \mu \mathrm{g})$ per lane were separated by $8 \%$ SDS-PAGE $(80 \mathrm{~V}$, $30 \mathrm{~min} ; 120 \mathrm{~V}, 90 \mathrm{~min}$ ) and then transferred to polyvinylidene fluoride membranes (Solarbio, Beijing, China). Membranes were incubated with rabbit anti-mouse SIRT1 monoclonal antibody (1:1,000 dilution) or rabbit anti-mouse Nrf2 polyclonal antibody (1:500 dilution) at $4^{\circ} \mathrm{C}$ for $24 \mathrm{~h}$ and washed with Tris-buffered saline containing Tween 20 (Solarbio). Subsequent to washing, samples were incubated with horseradish peroxidase-conjugated goat anti-rabbit immunoglobulin G secondary antibody (cat. no. sc-2030; 1:2,000 dilution; Santa Cruz Biotechnology, Inc., Dallas, TX, USA) for $1 \mathrm{~h}$ at room temperature and visualized using Western Blotting Luminol reagent (cat. no. sc-2048; Santa Cruz Biotechnology, Inc.). Images of the blots were captured using the ChemiDoc ${ }^{\mathrm{TM}}$ MP Imaging System and quantified by densitometry using Quantity One 4.52 software.

Enzyme-linked immunosorbent assay (ELISA). The heme oxygenase-1 (HO-1) activity in samples of the lower part of the left lung of six mice was measured by commercially available ELISA kits (Westang Biotechnology Co., Shanghai, China). ELISA was performed following the protocols provided by the manufacturer.

Detection of superoxide dismutase (SOD) and catalase (CAT) activity and protein levels of glutathione (GSH) and malondialdehyde (MDA). The MDA and GSH contents in samples of the lower part of the left lung of six mice were detected according to the instructions of the MDA and GSH assay kits purchased from Nanjing Jiancheng Bioengineering Research Institute (Nanjing, China). The SOD and CAT activities were detected according to the instructions of the SOD and CAT assay kits (Nanjing Keygen Biotech Co., Ltd., Nanjing, China).

Statistical analysis. All data are described as the mean \pm standard deviation and analyzed using SPSS 19.0 software (International Business Machines, Armonk, NY, USA). The differences between groups were analyzed by one-way analysis 
A
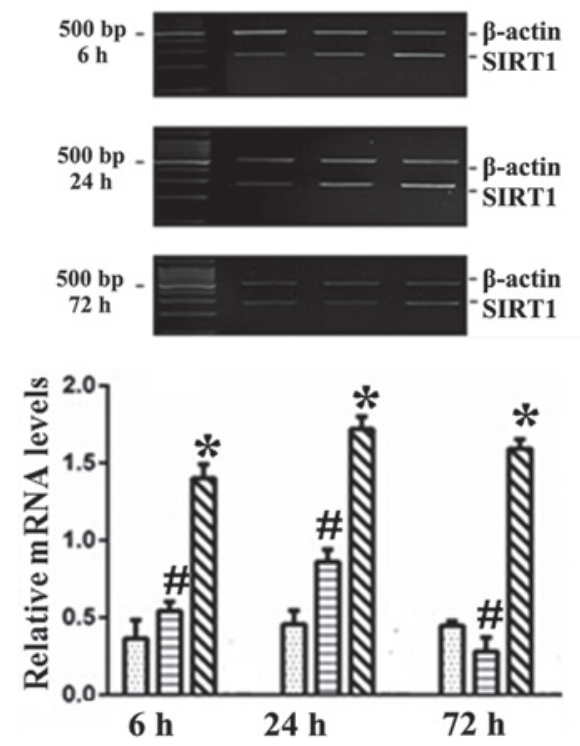

C
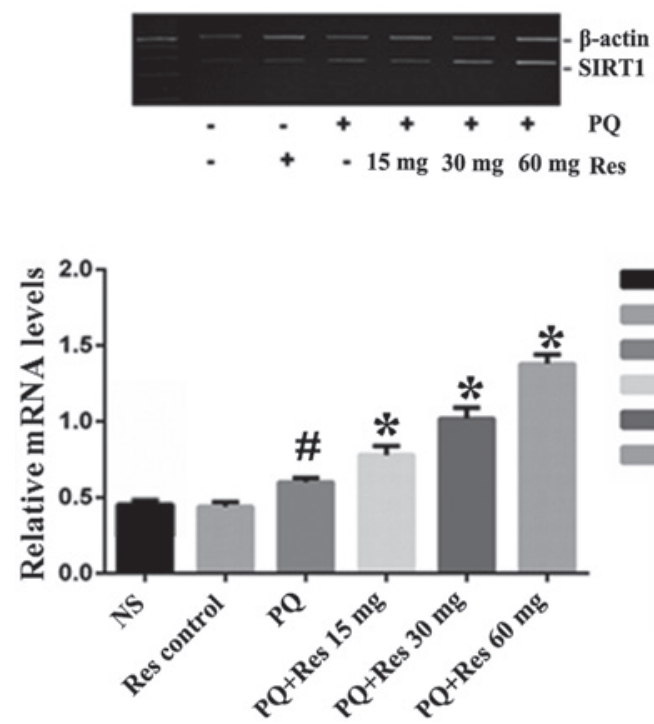

B
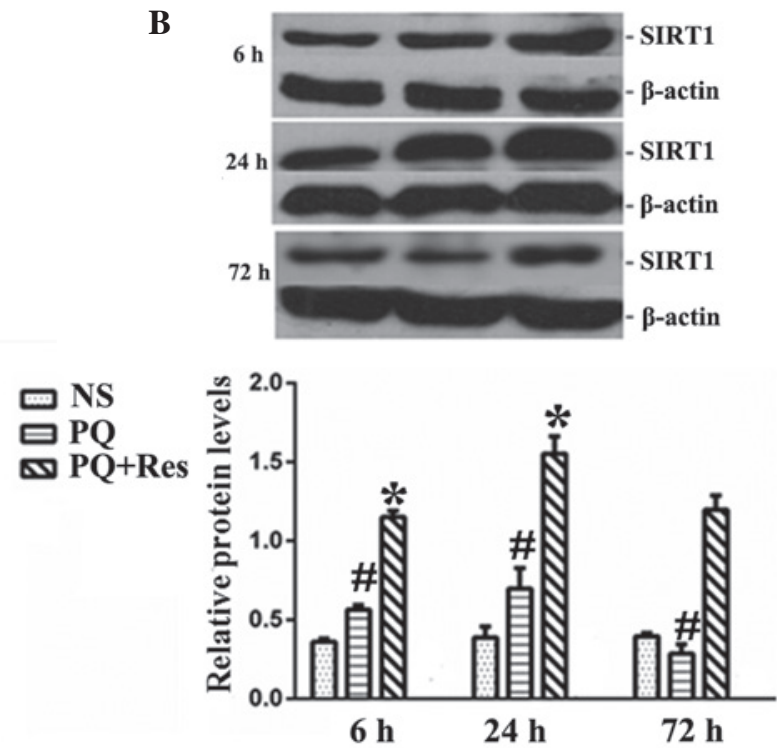

D
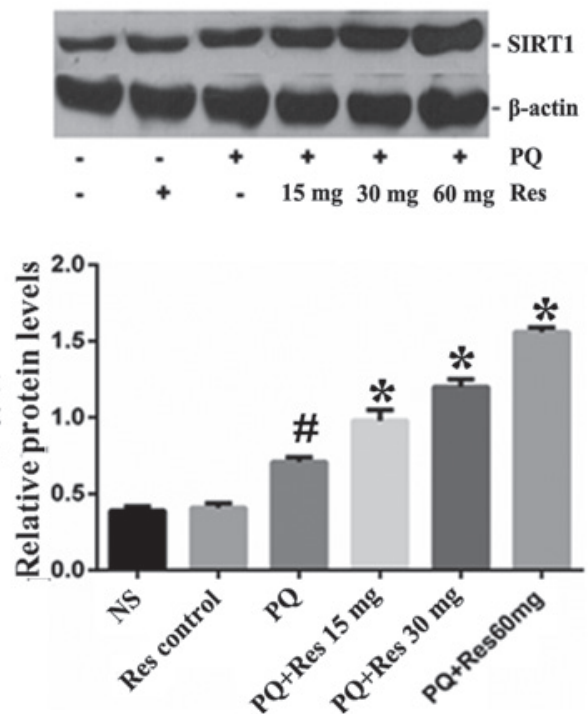

Figure 1. Res upregulated the expression of SIRT1 in PQ-induced mouse lung tissue. (A) mRNA and (B) protein levels of SIRT1 following 6-, 24- and 72-h PQ exposure. (C) mRNA and (D) protein levels of SIRT1 following 15, 30 and $60 \mathrm{mg}$ Res treatment. Data are presented as the mean \pm standard deviation (n=6). ${ }^{\#} \mathrm{P}<0.05$ vs. the NS group, ${ }^{\text {P }}<0.05$ vs. the $\mathrm{PQ}$ group. Res, resveratrol; SIRT1, sirtuin 1; PQ, paraquat; sqPCR, semiquantitative polymerase chain reaction; NS, normal saline.

of variance. $\mathrm{P}<0.05$ was considered to indicate a statistically significant difference.

\section{Results}

Res upregulated the expression of SIRT1 in $P Q$-exposed lung tissue. To assess the potential role of SIRT1 in PQ-induced lung injury, the SIRT1 levels in lung tissue prior and following Res injection were determined. As demonstrated in Fig. 1A and B, the expression of SIRT1 mRNA and protein levels were elevated at 6 and $24 \mathrm{~h}$ after PQ exposure compared with the saline group $(\mathrm{P}<0.05)$. At $72 \mathrm{~h}$, the SIRT1 mRNA and protein levels were significantly decreased compared with the saline group $(\mathrm{P}<0.05)$. Additionally, administration of $30 \mathrm{mg} / \mathrm{kg}$ Res significantly increased the mRNA and protein levels of SIRT1 at all time points compared with the PQ group $(\mathrm{P}<0.05)$. Administration of 15,30 or $60 \mathrm{mg} / \mathrm{kg}$
Res upregulated SIRT1 expression levels in the lung at $24 \mathrm{~h}$ compared with the $\mathrm{PQ}$ group $(\mathrm{P}<0.05$; Fig. $1 \mathrm{C}$ and $\mathrm{D})$.

Res attenuates $P Q$-induced lung injury in mice. Histopathological and W/D weight ratio analyses were performed to determine the effect of Res on PQ-induced lung injury. Compared with the control group, PQ administration caused marked lung hemorrhage, edema, alveolar septal thickening, influx of inflammatory cells and fibrin deposition (Fig. 2A). In the PQ+Res group, similar changes were identified, however to a lesser degree. Additionally, the W/D weight ratios of the lung tissues were significantly increased following PQ administration compared with the ratios in the NS group $(\mathrm{P}<0.05$; Fig. $2 \mathrm{~B})$. In the $\mathrm{PQ}+$ Res group, the lung W/D weight ratio was significantly lower than that in the PQ group at 24 and $72 \mathrm{~h}$ subsequent to $\mathrm{PQ}$ exposure $(\mathrm{P}<0.05$; Fig. 2B). 
A

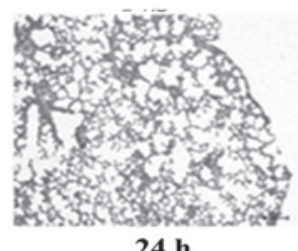

NS
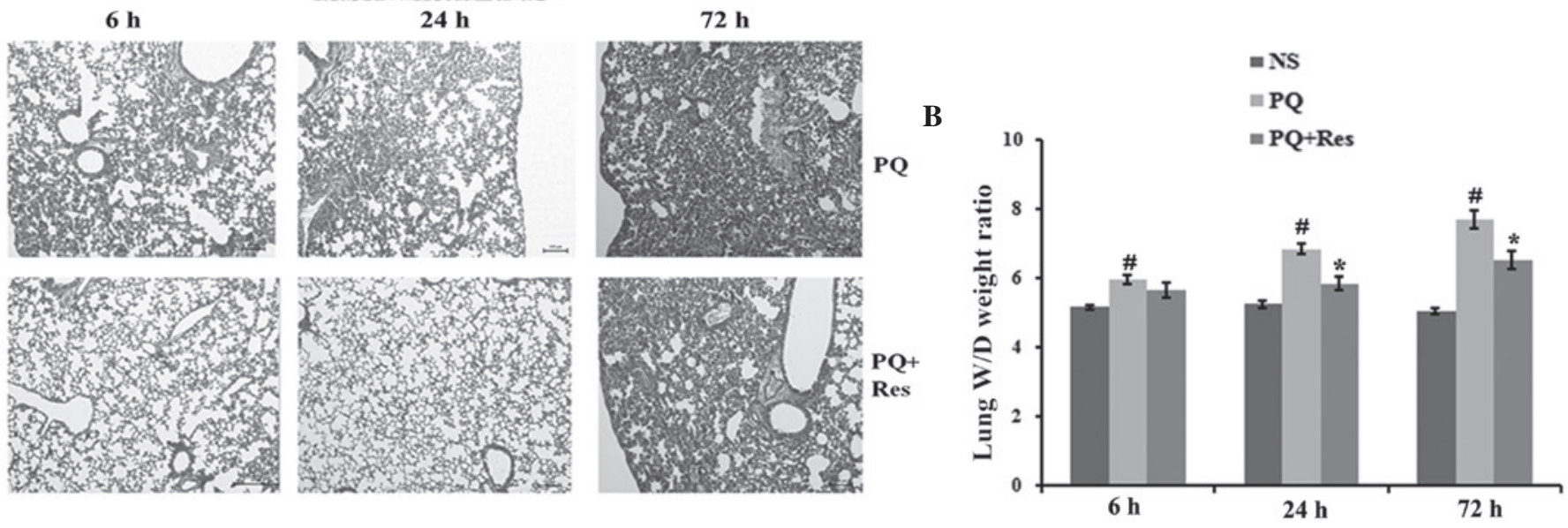

Figure 2. Res attenuates PQ-induced lung injury in mice. (A) Histological examination of mouse lung tissue was performed at 6,24 and $72 \mathrm{~h}$ post-PQ treatment. Tissue was fixed and stained with hematoxylin and eosin (magnification, x100). In the he NS group, parenchyma and lung airways are normal, whereas the PQ groups show marked lung hemorrhage, edema, alveolar septal thickening, influx of inflammatory cells and fibrin deposition. These changes were ameliorated by Res treatment. (B) The lung W/D ratio was determined at 6,24 and $72 \mathrm{~h}$ after PQ administration. Data are presented as the mean \pm standard deviation (n=6). ${ }^{\#} \mathrm{P}<0.05$ vs. the NS group, ${ }^{\mathrm{P}}<0.05$ vs. the $\mathrm{PQ}$ group. Res, resveratrol; $\mathrm{PQ}$, paraquat, W/D, wet/dry; NS, normal saline.

A

$6 \mathrm{~h}$

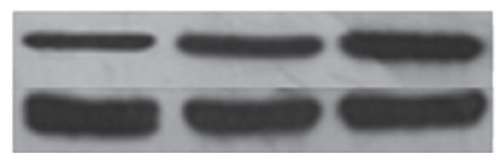

-NRF2

- GAPDH

$24 \mathrm{~h}$

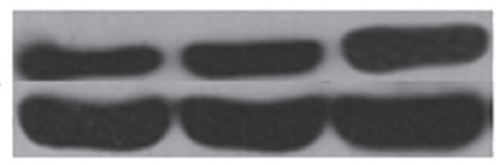

NRF 2

GAPDH

$72 \mathrm{~h}$

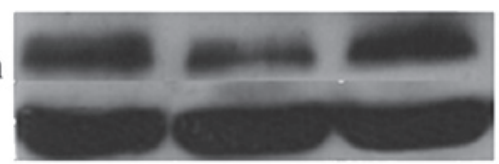

-NRF2

-GAPDH

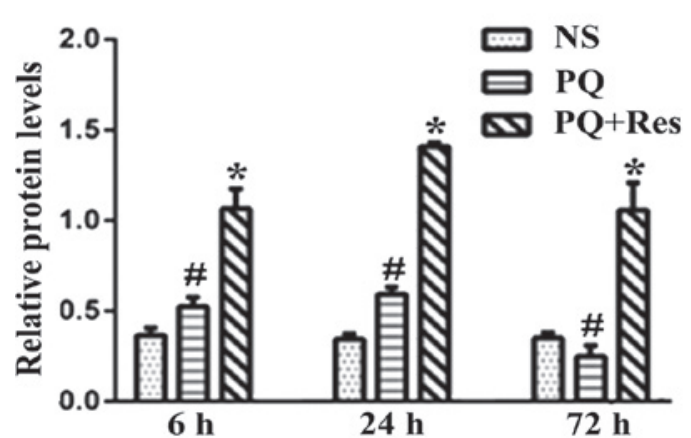

B
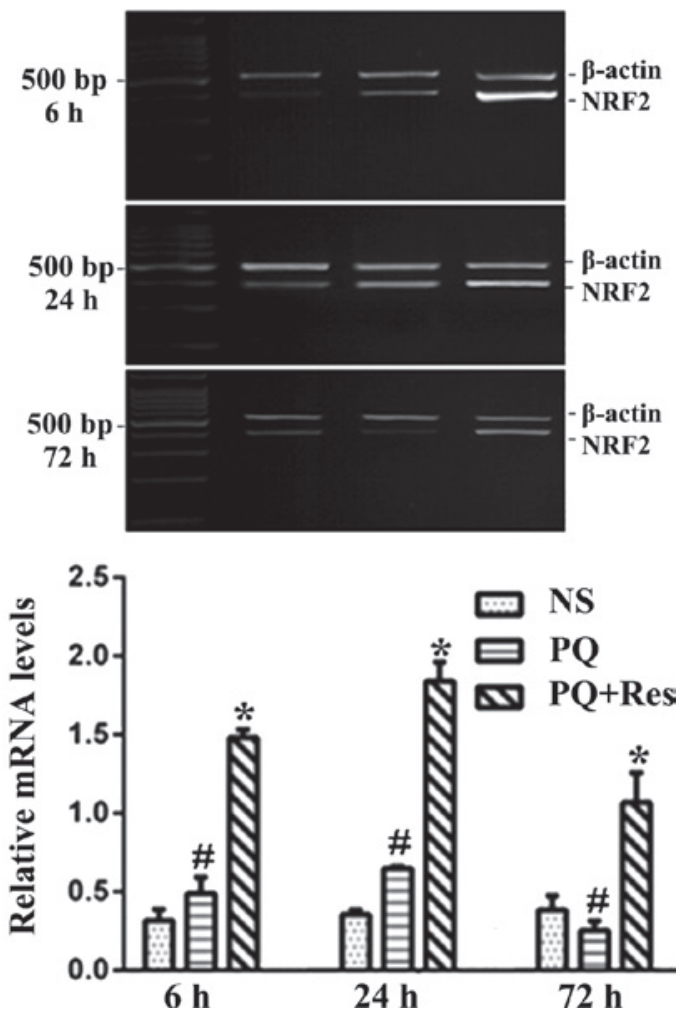

Figure 3. Effects of Res on NRF2 expression levels in mouse lung tissue following PQ exposure. The protein and mRNA expression levels of NRF2 were determined by (A) western blot and (B) semiquantitative polymerase chain reaction analyses, respectively, at 6, 24 and $72 \mathrm{~h}$ after PQ administration. Data are presented as the mean \pm standard deviation $(n=6) .{ }^{*} \mathrm{P}<0.05$ vs. the NS group, ${ }^{*} \mathrm{P}<0.05$ vs. the PQ group. Res, resveratrol; NRF2, nuclear factor, erythroid 2-like 2; PQ, paraquat, NS, normal saline.

Effects of Res on NRF2 expression in the lung following $P Q$ exposure. To investigate whether the protective role of Res was mediated through NRF2, the NRF2 expression levels in the mouse lung tissues were determined. NRF2 protein and mRNA expression levels were significantly elevated at 6 and $24 \mathrm{~h}$ after PQ administration but were decreased at $72 \mathrm{~h}$, compared 


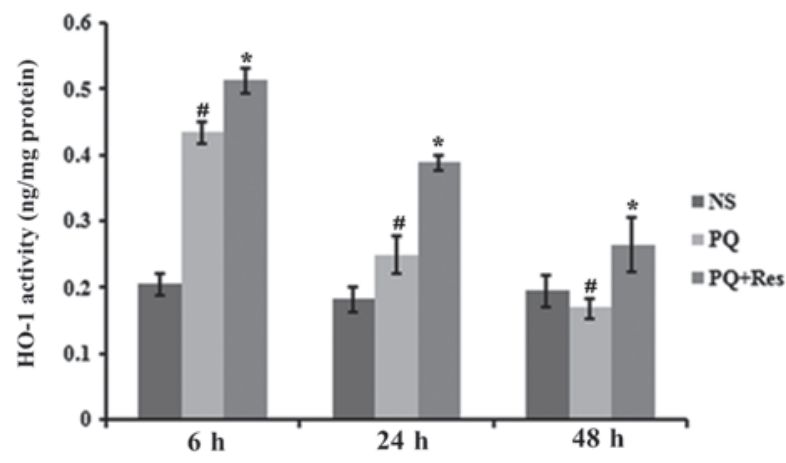

Figure 4. Effects of Res on HO-1 activity in mouse lung tissue after PQ exposure. The activity of HO-1 was determined by ELISA at 6, 24 and $72 \mathrm{~h}$ after PQ administration. Data are presented as the mean \pm standard deviation $(\mathrm{n}=6) .{ }^{*} \mathrm{P}<0.05$ vs. the NS group, ${ }^{*} \mathrm{P}<0.05$ vs. the $\mathrm{PQ}$ group. Res, resveratrol; HO-1, heme oxygenase-1; PQ, paraquat; NS, normal saline.
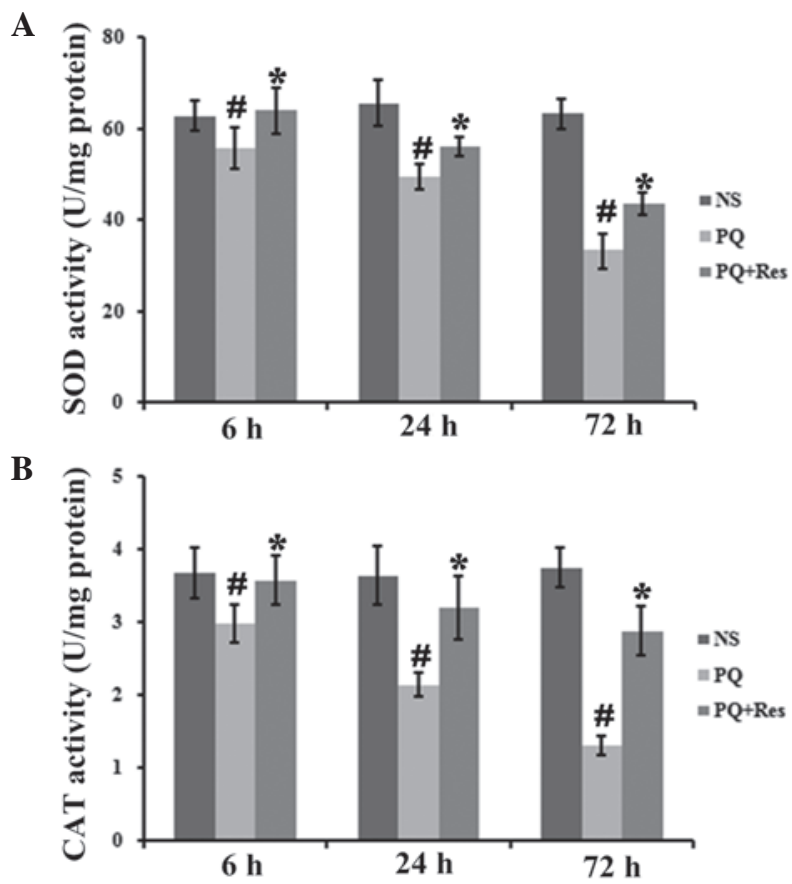

Figure 5. Effects of Res and PQ on SOD and CAT activity in mouse lung tissues. The activity of (A) SOD and (B) CAT were determined at 6, 24 and $72 \mathrm{~h}$ after $\mathrm{PQ}$ administration. Data are presented as the mean \pm standard deviation $(\mathrm{n}=6) .{ }^{~} \mathrm{P}<0.05$ vs. the NS group, ${ }^{*} \mathrm{P}<0.05$ vs. the $\mathrm{PQ}$ group. Res, resveratrol; $\mathrm{PQ}$, paraquat; SOD, superoxide dismutase; CAT, catalase; NS, normal saline.

with the NS group $(\mathrm{P}<0.05$; Fig. 3). However, Res administration significantly increased NRF2 protein expression levels compared with the $\mathrm{PQ}$ group at all time points ( $\mathrm{P}<0.05$; Fig. 3).

Effects of Res on HO-1 activity in mouse lung tissue following $P Q$ exposure. Compared with the NS group, HO-1 activity in the lung tissue was upregulated markedly at 6 and $24 \mathrm{~h}$ after PQ administration, but was decreased at $72 \mathrm{~h}(\mathrm{P}<0.05$; Fig. 4). In the $\mathrm{PQ}+$ Res group, HO-1 activity was upregulated compared with that in the PQ group (P<0.05; Fig. 4).

Effects of Res and PQ on SOD and CAT activity levels in mouse lung tissues. SOD and CAT activity levels in the mouse lung tissue were measured. Compared with the NS group, the PQ
A

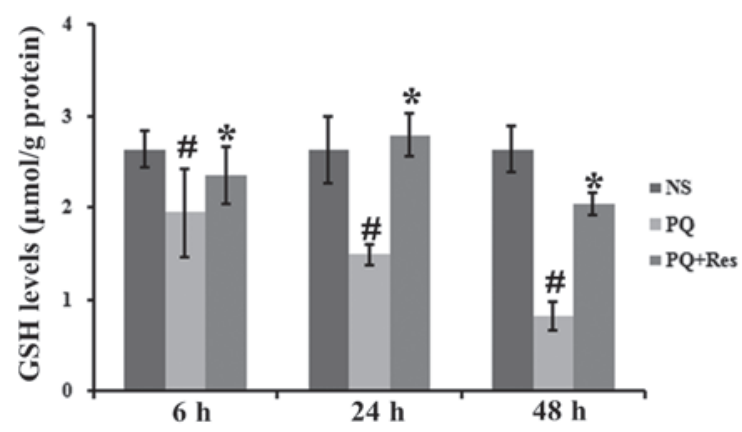

B

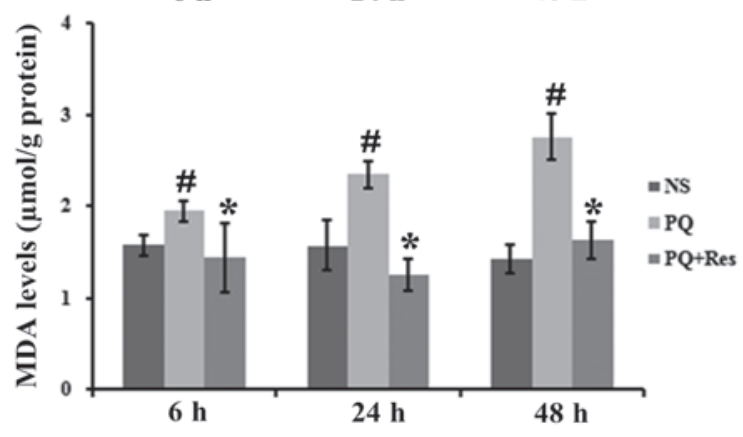

Figure 6. Effects of Res and PQ on (A) GSH and (B) MDA levels in mouse lung tissue. Levels of GSH and MDA were determined at 6, 24 and $72 \mathrm{~h}$ after PQ administration. Data are presented as the mean \pm standard deviation ( $\mathrm{n}=6)$. ${ }^{\text {"P}} \mathrm{P}<0.05$ vs. the NS group, ${ }^{*} \mathrm{P}<0.05$ vs. the PQ group. Res, resveratrol; PQ, paraquat; GSH, glutathione; MDA, malondialdehyde; NS, normal saline.

group exhibited a significant decrease in SOD and CAT activity levels at all time points $(\mathrm{P}<0.05$; Fig. 5). Following Res administration, SOD and CAT activity levels significantly increased in the lung tissue, compared with the NS group $(\mathrm{P}<0.05$; Fig. 5).

Effects of Res and $P Q$ on GSH and MDA levels in mouse lung tissues. Following PQ administration, GSH protein levels were decreased in the lung tissue compared with the NS group $(\mathrm{P}<0.05$; Fig. 6A). The GSH protein levels were significantly increased in the $\mathrm{PQ}+$ Res group compared with those in the PQ group ( $\mathrm{P}<0.05$; Fig. 6A). By contrast, $\mathrm{PQ}$ administration led to an increase in MDA activity levels compared with the NS group $(\mathrm{P}<0.05$; Fig. 6B). Res administration decreased MDA activity levels compared with those in the $\mathrm{PQ}$ group $(\mathrm{P}<0.05$; Fig. 6B).

\section{Discussion}

The lung is targeted in PQ poisoning through the pulmonary polyamine uptake system that recruits $P Q$. This results in a 6-10-fold increase in the lung PQ levels compared with plasma levels. Based on its induction of redox cycling, PQ in the lung results in oxidative stress-associated cell death and lung injury $(14,15)$.

Sirtuins are a unique class of type III histone deacetylases with seven isoforms, SIRT1-7 (16). SIRT1 is the best characterized member among the mammalian sirtuins (16). Previous studies have revealed a crosstalk between the SIRT1 expression levels and oxidative stress $(5,17)$. Oxidative stress can inhibit the SIRT1 expression, and the overexpression of SIRT1 has been demonstrated to inhibit apoptosis induced by oxidative stress $(18,19)$. The current study demonstrated increased SIRT1 expression levels in mouse lung tissue at 6 and $24 \mathrm{~h}$ after PQ 
administration. However, long-term exposure to PQ significantly decreased the expression of SIRT1. Treatment with Res, a known allosteric activator of SIRT1, upregulated the expression of SIRT1, accompanied by decreased oxidative stress and lung injury. This demonstrated that the SIRT1 agonist in Res treatment has a protective role in PQ-induced lung injury by decreasing oxidative stress.

A major mechanism in the cellular defense against oxidative stress is the activation of the NRF2 antioxidant response element signaling pathway (20). Previous studies have demonstrated that NRF2 is important in PQ-induced lung injury; PQ can inhibit the NRF2 expression in vitro and in vivo $(6,21)$. Additionally, overexpression of NRF2 can ameliorate PQ-induced lung injury and cell apoptosis $(21,22)$. The function of the NRF2-antioxidant pathway is controlled by multiple factors, including the acetylation-deacetylation of NRF2. Kawai et al (7) demonstrated that SIRT1-mediated deacetylation of the NRF2 protein terminated the transcription of antioxidant genes in vitro. However, other studies have demonstrated that SIRT1 overexpression significantly promoted the nuclear accumulation, DNA binding and transcriptional activity of NRF2 and NRF2-mediated gene expression $(8,9,23)$. In the current study, an increase in the SIRT1 expression levels by Res was associated with a high level of NRF2. In addition, the HO-1, SOD and CAT activity and the levels of GSH were upregulated following Res administration. Indeed, previous studies indicated that the acetylation of NRF2 can reduce NRF2 stability and impaired antioxidant defenses (24). Therefore, as a protein deacetylase, SIRT1 may be important for NRF2 protein stability and expression.

Taken together, the results of the current study demonstrated that Res, an SIRT1 activator, can reduce PQ-induced lung injury by regulating SIRT1 expression in combination with the NRF2 antioxidant pathway. The present study indicated that SIRT1 and NRF2 serve a critical role in PQ-induced lung injury. However, the mechanism of regulation of NRF2 expression levels by SIRT1 requires further investigation.

\section{Acknowledgements}

This work was supported by the State Key Program of the Natural Science Foundation of Zhejiang province (no. LZ12H26001) and the Medical and Health Research Program of Zhejiang province (no. 2012ZDA034).

\section{References}

1. Gawarammana IB and Buckley NA: Medical management of paraquat ingestion. Br J Clin Pharmacol 72: 745-757, 2011.

2. Blanco-Ayala T, Andérica-Romero AC and Pedraza-Chaverri J: New insights into antioxidant strategies against paraquat toxicity. Free Radic Res 48: 623-640, 2014.

3. Hong GL, Liu JM, Zhao GJ, Wang L, Liang G, Wu B, Li MF, Qiu QM and Lu ZQ: The reversal of paraquat-induced mitochondria-mediated apoptosis by cycloartenyl ferulate, the important role of Nrf2 pathway. Exp Cell Res 319: 2845-2855, 2013.

4. Caito S, Rajendrasozhan S, Cook S, Chung S, Yao H, Friedman AE, Brookes PS and Rahman I: SIRT1 is a redox-sensitive deacetylase that is post-translationally modified by oxidants and carbonyl stress. FASEB J 24: 3145-3159, 2010.

5. Salminen A, Kaarniranta K and Kauppinen A: Crosstalk between Oxidative Stress and SIRT1: Impact on the Aging Process. Int J Mol Sci 14: 3834-3859, 2013.
6. Hong GL, Cai QQ, Tan JP, Jiang XZ, Zhao GJ, Wu B, Li MF, Qiu QM and Lu ZQ: Mifepristone-inducible recombinant adenovirus attenuates paraquat-induced lung injury in rats. Hum Exp Toxicol 34: 32-43, 2015.

7. Kawai Y, Garduño L, Theodore M, Yang J and Arinze IJ: Acetylation-deacetylation of the transcription factor Nrf2 (nuclear factor erythroid 2-related factor 2) regulates its transcriptional activity and nucleocytoplasmic localization. J Biol Chem 286: 7629-7640, 2011.

8. Huang K, Huang J, Xie X, Wang S, Chen C, Shen X, Liu P and Huang H: Sirt1 resists advanced glycation end products-induced expressions of fibronectin and TGF- $\beta 1$ by activating the Nrf2/ARE pathway in glomerular mesangial cells. Free Radic Biol Med 65: 528-540, 2013.

9. Huang K, Chen C, Hao J, Huang J, Wang S, Liu P and Huang H: Polydatin promotes Nrf2-ARE anti-oxidative pathway through activating Sirt1 to resist AGEs-induced upregulation of fibronetin and transforming growth factor- $\beta 1$ in rat glomerular messangial cells. Mol Cell Endocrinol 399: 178-189, 2015.

10. Leonard SS, Xia C, Jiang BH, Stinefelt B, Klandorf H, Harris GK and Shi X: Resveratrol scavenges reactive oxygen species and effects radical-induced cellular responses. Biochem Biophys Res Commun 309: 1017-1026, 2003.

11. Borra MT, Smith BC and Denu JM: Mechanism of human SIRT1 activation by resveratrol. J Biol Chem 280: 17187-17195, 2005.

12. Novelle MG, Wahl D, Diéguez C, Bernier M and de Cabo R: Resveratrol supplementation: Where are we now and where should we go? Ageing Res Rev 21: 1-15, 2015.

13. Rivera H, Shibayama M, Tsutsumi V, Perez-Alvarez V and Muriel P: Resveratrol and trimethylated resveratrol protect from acute liver damage induced by $\mathrm{CCl} 4$ in the rat. J Appl Toxicol 28: 147-155, 2008

14. Hoet PHM and Nemery B: Polyamines in the lung: Polyamine uptake and polyamine-linked pathological or toxicological conditions. Am J Physiol Lung Cell Mol Physiol 278: L417-L433, 2000.

15. Dinis-Oliveira RJ, Pontes H, Bastos ML, Remião F, Duarte JA and Carvalho F: An effective antidote for paraquat poisonings: The treatment with lysine acetylsalicylate. Toxicology 255 : 187-193, 2009.

16. Cantó $\mathrm{C}$ and Auwerx J: Targeting sirtuin 1 to improve metabolism: All you need is NAD(+)? Pharmacol Rev 64: 166-187, 2012.

17. Alcendor RR, Gao S, Zhai P, Zablocki D, Holle E, Yu X, Tian B, Wagner T, Vatner SF and Sadoshima J: Sirt1 regulates aging and resistance to oxidative stress in the heart. Circ Res 100: 1512-1521, 2007.

18. Cao C, Lu S, Kivlin R, Wallin B, Card E, Bagdasarian A, Tamakloe T, Wang WJ, Song X, Chu WM, et al: SIRT1 confers protection against UVB- and $\mathrm{H} 2 \mathrm{O} 2$-induced cell death via modulation of p53 and JNK in cultured skin keratinocytes. J Cell Mol Med 13 (9B): 3632-3643, 2009.

19. Hasegawa K, Wakino S, Yoshioka K, Tatematsu S, Hara Y, Minakuchi H, Washida N, Tokuyama H, Hayashi K and Itoh H: Sirt1 protects against oxidative stress-induced renal tubular cell apoptosis by the bidirectional regulation of catalase expression. Biochem Biophys Res Commun 372: 51-56, 2008.

20. Cho HY, Reddy SP and Kleeberger SR: Nrf2 defends the lung from oxidative stress. Antioxid Redox Signal 8: 76-87, 2006.

21. He XY, Zhao GJ, Lu ZQ, Hong GL, He F, Liang H, Qiu QM and Li JR: Oxidative stress of acute paraquat poisoned rats and sodium dimercaptopropane sulfonate intervention. Chin J Ind Hyg Occup Dis 27:476-479, 2009.

22. Jiang XZ, Song Q, Xu XP, Cai QQ, Hong GL, Liang H and Lu ZQ: The effects of Nrf2 gene expression induced by RU486 at different doses on A549 cell damage induced by paraquat. Chin J Ind Hyg Occup Dis 30: 268-272, 2012.

23. Yang Y, Li W, Liu Y, Sun Y, Li Y, Yao Q, Li J, Zhang Q, Gao Y, Gao L, et al: Alpha-lipoic acid improves high-fat diet-induced hepatic steatosis by modulating the transcription factors SREBP-1, FoxO1 and Nrf2 via the SIRT1/LKB1/AMPK pathway. J Nutr Biochem 25: 1207-1217, 2014.

24. Mercado N, Thimmulappa R, Thomas CM, Fenwick PS, Chana KK, Donnelly LE, Biswal S, Ito K and Barnes PJ: Decreased histone deacetylase 2 impairs Nrf2 activation by oxidative stress. Biochem Biophys Res Commun 406: 292-298, 2011. 